Enferm Bras 2021;20(4):549-63

doi: $10.33233 /$ eb.v20i4.4328

\title{
REVISÃO
}

\section{Relação entre depressão e desnutrição em idosos}

Mylena de Souza Ribas*, Jessica de Almeida Rodrigues*, Jéssica Cristina Santana de Sousa*, Thiálita Rebeca Oliveira de Castro*, Marcos Vinicius da Silva*, Beatriz Andrade dos Santos*, Maria de Jesus Oliveira*, Vanessa Alvarenga Pegoraro, M.Sc.

${ }^{\star}$ Estudante de graduação Enfermagem do UNICEUB, ${ }^{* *}$ Professora Assistente do Curso de Enfermagem FACES/UNICEUB

Recebido em 11 de fevereiro de 2021; Aceito em 25 de agosto de 2021.

Correspondência: Vanessa Alvarenga Pegorato, FACES/UNICEUB, 707/907 Campus Universitário, SEPN, Asa Norte, 70790-075 Brasília DF

\author{
Mylena de Souza Ribas: mylena.ribas@gmail.com \\ Jessica de Almeida Rodrigues: jessica.almeida@sempreceub.com \\ Jéssica Cristina Santana de Sousa: jessika_santana08@hotmail.com \\ Thiálita Rebeca Oliveira de Castro: thialitacastro@gmail.com \\ Marcos Vinicius da Silva: marcosxt21@gmail.com \\ Beatriz Andrade dos Santos: beatrizandrade280197@gmail.com \\ Maria de Jesus Oliveira: maria.2382@outlook.com \\ Vanessa Alvarenga Pegoraro: vanessa.pegoraro@ceub.edu.br
}

\section{Resumo}

Introdução: Como as taxas de sobrevida estão elevadas, as doenças crônicas degenerativas são as enfermidades que mais acometem os idosos e observa-se que a desnutrição possui estreita relação com as diversas patologias, levando a uma má qualidade de vida. Objetivo: $O$ objetivo do presente artigo foi identificar na literatura a relação entre a depressão e a desnutrição em idosos. Métodos: Trata-se de um estudo bibliográfico, descritivo do tipo de revisão integrativa e abordagem qualitativa. Foram selecionados 10 artigos, nas bases de dados Medline, Scielo, Lilacs e Pubmed. Resultados: Conforme semelhança dos dados, foram criadas duas categorias: "Suscetibilidade do gênero feminino na associação entre depressão e desnutrição" e "A relação entre depressão e desnutrição". A prevalência da depressão em mulheres é duas vezes mais que em homens, considerando fatores fisiológicos, hormonais, 
aspectos socioculturais. Conclusão: A depressão pode levar ao dentrimento do estado nutricional e a desnutrição pode ocasionar a depressão.

Palavras-chave: desnutrição; depressão; idoso.

\section{Abstract}

\section{Relationship between depression and malnutrition in the elderly}

Introduction: As survival rates are high, chronic degenerative diseases are the diseases that most affect the elderly and malnutrition is closely related to the various pathologies, leading to a poor quality of life. Aim: Therefore, the aim of this article was to identify in the literature the relationship between depression and malnutrition in the elderly. Methods: This is a descriptive, integrative review with qualitative approach. Ten articles were selected from the Medline, Scielo, Lilacs and Pubmed databases. Results: According to the similarity of the data, two categories were created: "Female susceptibility in the association between depression and malnutrition" and "The relationship between depression and malnutrition". The prevalence of depression in women is twice as high as in men, considering physiological, hormonal and socio-cultural factors. Conclusion: Depression can lead to a deterioration in nutritional status and malnutrition can cause depression.

Keywords: malnutrition; depression; aged.

\section{Resumen}

\section{Relación entre depresión y malnutrición en personas mayores}

Introducción: Como las tasas de supervivencia son altas, las enfermedades degenerativas crónicas son las enfermedades que más afectan a los ancianos y la desnutrición está estrechamente relacionada con las diversas patologías, lo que conduce a una mala calidad de vida. Objetivo: Por lo tanto, el objetivo de este artículo fue identificar en la literatura la relación entre la depresión y la desnutrición en los mayores. Métodos: Este es un estudio bibliográfico, descriptivo del tipo de revisión integradora y enfoque cualitativo. Se seleccionaron 10 artículos de las bases de datos Medline, Scielo, Lilacs y Pubmed. Resultados: De acuerdo con la similitud de los datos, se crearon dos categorías: la susceptibilidad femenina en la asociación entre depresión y desnutrición y la relación entre depresión y desnutrición. La prevalencia de la depresión en las mujeres es el doble que en hombres, considerando aspectos fisiológicos, hormonales y socioculturales. Conclusión: La depresión puede conducir a un deterioro en el estado nutricional y la desnutrición puede causar depresión.

Palabras-clave: desnutrición; depresión; anciano. 
Introdução

O envelhecimento populacional crescente ocorre devido a mudanças nos indicadores da saúde, como a redução da taxa de mortalidade, redução da fecundidade e aumento da expectativa de vida [1].

Esse fenômeno começou a ser melhor observado no Brasil, principalmente na década de 1970, quando os levantamentos demográficos mostraram a proporção da população idosa em detrimento das crianças, surgindo assim a transição demográfica. No século passado, a população falecia com doenças infectocontagiosas, pela deficiência dos recursos necessários para os cuidados com a saúde. A partir de vários estudos e sobretudo do surgimento de tecnologias para a saúde, atualmente são as doenças crônicas degenerativas que acometem a população idosa [2].

Com a evolução do envelhecimento, a organização das políticas públicas voltadas para as necessidades da saúde da população idosa se mostra despreparada, o que aumenta a probabilidade de obterem limitações funcionais e cognitivas [3]. Dessa forma, é fundamental proporcionar à população uma senescência saudável e com melhora na qualidade de vida para não viverem com aflição com as possíveis limitações, dependências e incapacidades [4].

O transtorno depressivo é a doença que mais afeta os idosos. Acredita-se que em 2030 ocorrerá um aumento absoluto de brasileiros com 60 anos ou mais excedendo o de crianças de 0 a 14 anos, e espera-se que em 2050 atingirá 38 milhões de idosos com depressão [5]. A cada ano, a depressão é a enfermidade mais frequente nessa população específica, e eleva a morbidade e a mortalidade, requerendo mais atenção da saúde pública [6].

A depressão é determinada como uma patologia multifatorial abordando áreas do humor e afeto, que efetua grande impacto funcional, envolvendo aspectos biológicos, psicológicos e sociais [3]. O fator desencadeante mais significativo para a depressão é a perda de algo muito importante, dificuldades no diálogo, isolamento social e problemas financeiros. O idoso está mais propenso a obter essa enfermidade, por frequentemente passar por mudanças ou perdas [1,7].

A partir do supracitado, observa-se que os idosos com condições econômicas baixas, que na vida passaram por estresse excessivo marcante, idosos com baixa qualidade de vida, situações de saúde reduzidas e que possuem limitações funcionais ou que já desenvolveram alguma doença crônica são mais suscetíveis a desenvolverem a depressão [8].

Os idosos que vivem com limitações cognitivas e funcionais possuem de forma reduzida a possibilidade de realizarem a sua própria autonomia e, consequentemente, 
as suas necessidades básicas da vida diária ficam comprometidas, como higiene e alimentação [9]. Eles podem apresentar diminuição na habilidade de manuseio e conhecimento reduzido dos alimentos, dificuldades na própria mastigação e deglutição, aumentando o risco de desnutrição [10].

A desnutrição pode estar diretamente relacionada com a depressão, pois além de proporcionar uma má qualidade de vida do idoso, predispondo-o a diversas manifestações negativas que interferem na sua vida, como irritabilidade, ansiedade e até mesmo a depressão. Dessa maneira, a desnutrição proteico-energético de acordo com alguns estudos está sendo associada ao desenvolvimento da disfunção cognitiva [9].

A desnutrição ou má nutrição é caracterizada por um estado nutricional no qual existe uma ingestão insuficiente de energia, micronutrientes e proteínas que tem como resultado uma interação profunda entre a sua alimentação, estado de saúde, aspectos econômicos e condições sociais. Logo, percebe-se a necessidade de avaliação e conhecimento do estado nutricional, das modificações psicológicas, corporais e até mesmo sociais. E para a avaliação do estado nutricional dos idosos, deve-se aplicar a Miniavaliação Nutricional (MNA), considerado um método básico e fácil de utilização para detecção da desnutrição. Além disso, é possível avaliar os aspectos físicos e mentais que são fatores que interferem na maioria das vezes o estado nutricional [11].

Dessa forma, percebe-se a importância em também realizar uma identificação dos idosos desnutridos, pois obtém uma associação com diversas complicações que afetam diretamente a vida do idoso, dentre elas a redução da capacidade mental, o que ocasiona um aumento maior da assistência de saúde, portanto, a desnutrição precisa ser compreendida na avaliação da depressão [12].

Ao observar que ao longo dos anos o envelhecimento populacional está aumentando de forma abrupta e a população sendo acometida por doenças crônicas como a depressão, existe uma enorme necessidade de identificar precocemente e discutir sobre a sua importância por possuir grande prevalência em várias idades, principalmente em idosos [13]. Deve-se ter um olhar holístico para fazer a identificação adequada do estado nutricional por ser um importante fator para o funcionamento adequado do organismo, visando o tratamento para melhorar a qualidade de vida do idoso e observar como interfere no estado cognitivo dessa população [14].

Assim, o presente estudo teve como objetivo identificar na literatura a relação entre a depressão e a desnutrição em idosos. 
Trata-se de um estudo bibliográfico, descritivo do tipo de revisão integrativa e abordagem qualitativa. Realizou-se o estudo a partir das seguintes etapas: 1) Identificação do tema e seleção da questão de pesquisa; 2) Estabelecimento de critérios para inclusão e exclusão de estudos na literatura; 3) Definição das informações a serem extraídas dos estudos selecionados; 4) Avaliação dos estudos incluídos; 5) Interpretação dos resultados e 6) Apresentação da revisão/síntese do conhecimento [15].

Estabeleceu-se como quesito primordial que contribuiu para o desenvolvimento da pesquisa a seguinte questão: Qual a relação da desnutrição e depressão nos idosos? Realizou-se uma busca bibliográfica nas bases de dados eletrônicas: Medline (Medical Literature Analysis and Retrieval System Online), Scientific Electronic Library Online (Scielo), Literatura Latino-Americana e do Caribe de Informações em Ciências da Saúde (Lilacs), Pubmed, nos meses de março a abril de 2020.

Utilizaram-se os seguintes descritores indexados no DeCS: "Desnutrição", "depressão", “idoso". Possibilitou-se com esses descritores, a realização de três cruzamentos, inseridos nas bases de dados selecionadas: 1) Idoso AND Desnutrição AND Depressão; 2) Idoso AND Desnutrição; 3) Desnutrição AND Depressão.

A leitura dos títulos, resumos e textos completos foi realizada por duas pessoas, de forma independente e os resultados foram comparados com intuito de certificar os critérios de elegibilidade. Captaram-se, no que diz respeito ao recorte temporal, todas as publicações disponíveis em cada base de dados no período dos últimos dez anos. Elencaram-se como critérios de inclusão: artigos completos, disponíveis gratuitamente nas bases de dados selecionadas, que abordam as associações entre desnutrição e depressão no idoso, escritos em inglês, espanhol e português. E critérios de exclusão: publicações que não abordassem sobre desnutrição e depressão em idosos, artigos pagos e com delimitação temporal com mais de dez anos em formato editoriais, resumos, teses, editoriais e carta de opinião.

Coletaram-se os dados por meio das informações contidas no instrumento elaborado pelos autores, que continham questionamentos sobre: identificação do estudo (título do artigo, título do periódico, autores, país, idioma e ano de publicação) tipo de publicação características metodológicas do estudo (objetivo, abordagem da pesquisa, amostra, resultados, análise, implicações e nível de evidência) [16].

Avaliaram-se os títulos dos artigos encontrados, selecionaram-se aqueles que possuíam vinculação com o objetivo desta pesquisa, em seguida foi realizada uma análise crítica e compreensiva dos resumos, atentando para os critérios de inclusão. Logo após leram-se completamente todos os textos selecionados que demonstraram resposta à pergunta norteadora. 
A seleção dos artigos pode ser representada através de fluxograma com as recomendações do PRISMA na figura 1 [17].

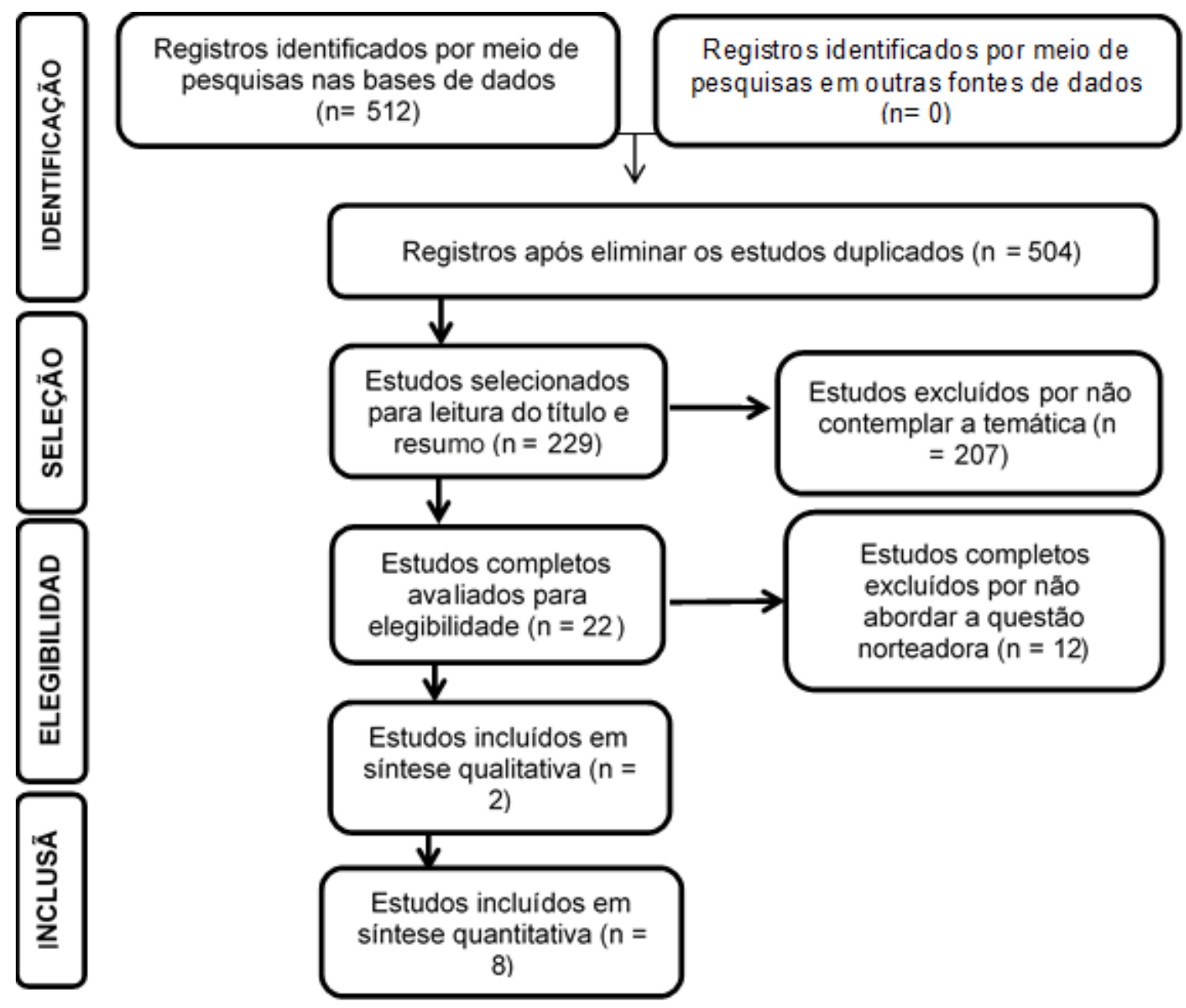

Fonte: autoras, 2020.

Figura 1 - Fluxograma de seleção dos estudos. Adaptação do PRISMA (2015), Brasília (DF), Brasil, 2020

\section{Resultados}

Foram encontradas 512 referências, sendo 134 artigos na base de dados da Medline utilizando três descritores: idoso AND desnutrição AND depressão, 15 artigos Lilacs utilizando três descritores: idoso AND desnutrição AND depressão, 41 na Scielo com dois descritores: idoso AND desnutrição e 130 no Pubmed, com dois descritores: idoso AND desnutrição e 192 artigos com dois descritores: desnutrição AND depressão. No Banco de Dados Enfermagem (BDEnf), após colocação de todos os descritores utilizados para a pesquisa, não foi encontrado nenhum resultado. 
Após a leitura criteriosa do título e resumo, foram excluídos aqueles que não estavam de acordo com o objetivo proposto, que fugiam da pergunta norteadora e os artigos duplicados.

No total foram selecionados para o desenvolvimento do trabalho apenas 10 artigos que foram apresentados os títulos, tipo de estudos, periódico, bases de dados encontradas e principais achados no (quadro 1).

Quadro 1 - Descrição dos artigos selecionados para revisão integrativa (ver PDF anexo)

Ao analisar criteriosamente os 10 artigos, foram listados os principais achados. Ao longo das buscas dos estudos nas bases de dados, observou-se o interesse dos autores sobre a temática, que ocorreu nos anos de 2013 a 2018. Verifica-se em 2013 apenas uma publicação; 2014 uma publicação; 2015 cinco publicações e 2018 três publicações. Desta forma observou-se que em $2015(n=5)$ e $2018(n=3)$ houve um maior registro de publicações.

Ressalta-se que, quanto ao método adotado nos estudos selecionados, o mais encontrado foi o método transversal, visto em nove artigos (Quadro 2 e figura 2). Tais dados nos demonstram que há uma escassez de publicações que abordem a associação da desnutrição com a depressão em idosos.

Algo que pode ter influenciado o aumento no quantitativo sobre a temática em 2015, foi que a OMS (Organização Mundial de Saúde) em 2015, realizou a publicação do Relatório Mundial de Envelhecimento e Saúde, que visa abordar estratégias que possam ser traçadas para a ação pública frente a longevidade que cresce cada vez mais e para que isso se fortaleça há uma necessidade de cuidar melhor da saúde da população.

Quadro 2 - Distribuição dos estudos selecionados por nível de evidência [16]

\begin{tabular}{lc}
\multicolumn{1}{c}{ Nivel de evidência } & Quantidade \\
\hline Nivel 1 - revisão integrativa ou metanálise & 1 \\
Nivel 2- estudo observacional e transversal & 0 \\
Nivel 3 - estudo analítico, transversal e quantitativo & 0 \\
Nivel 4- estudo analítico, observacional com corte transversal, quantitativo & 1 \\
Nivel 5 - revisão sistemática de estudos qualitativos e descritivos & 1 \\
Nivel 6 - estudo quantitativo, descritivo, transversal & 4 \\
Nivel 7- estudo transversal quantitativo_ou quantitativo e transversal & 3 \\
\hline
\end{tabular}

Elaborada pelas autoras, 2020.

Dentre os artigos previamente selecionados, seguindo os critérios de inclusão e exclusão definidos para a revisão deste estudo, identificaram-se duas categorias para discussão: "Suscetibilidade do gênero feminino na associação entre depressão e desnutrição" e "A relação entre depressão e desnutrição". 
Discussão

Suscetibilidade do gênero feminino na associação entre depressão e desnutrição

As condições de depressão e desnutrição são mais favoráveis em ocorrer em mulheres, porque a depressão pode ser ocasionada por imagem corporal incorreta e dieta inadequada para perda de peso e/ou distúrbios alimentares frequentes entres mulheres [18].

As idosas desnutridas são mais vulneráveis a manifestar a depressão. Existe uma associação entre a desnutrição e sexo feminino, através do baixo grau de escolaridade, nível socioeconômico reduzido, viúvas, mulheres que moram sozinhas e que são dependentes de familiares [19]. Porém, não se encontraram aspectos demográficos que confirmassem a relação com a desnutrição, mas acredita-se que idosas são mais propensas a desenvolver risco de desnutrição, por considerar que o envelhecimento desenvolve mudanças nas composições corporais, elevando a desnutrição no avançar da idade [20].

As mulheres podem apresentar manifestações depressivas decorrentes de experiências passadas, que são fatores que podem influenciar a depressão, como violência doméstica, estresse, ansiedade, obrigações de funções, sentimento à experiências traumáticas vividas seja na infância e adolescência, perda de entes queridos e viuvez [21].

Conforme a pesquisa realizada no ambulatório de um hospital público de referência de alta especialidade na cidade do México, com 114 pessoas avaliadas, a desnutrição e depressão ocorreu em uma porcentagem maior no sexo feminino, porém o sexo masculino ao ser calculado, percebeu-se nestes um maior risco para desenvolver desnutrição. De acordo com os autores, vários estudos apontam que mulheres possuem prevalência maior de risco para desnutrição e depressão [22].

Relação entre desnutrição e depressão

De acordo com os autores, existem diversos fatores específicos condicionantes para a perda de peso pela ingestão insuficiente de nutrientes, proveniente de aspectos psicólogicos incluindo a depressão e demência, situações sociais e médicas [19,23].

A problematização na qual esses fatores estão envolvidos é que na maioria das vezes estão associados ao consumo alimentar reduzido, tornando os idosos mais vulneráveis nutricionalmente, bem como as condições neuropsicológicas, que são 
designadas como um dos fatores importantes para desenvolver a desnutrição na população idosa [23].

Ainda existe uma incerteza na relação entre depressão e desnutrição, porém houve uma observância que nos idosos deprimidos tem percebido deficiências de vitaminas, como ácido fólico ou piridoxina, e alguns mineirais. Neste sentido, realizaram um estudo a fim de observar se há a diminuição dos sintomas depressivos com suplementos vitamínicos variados, utilizando ácido fólico e vitamina B12, entre outros, mas ainda assim os resultados se mostraram contraditórios [19,23].

Há uma importância significativa nos estudos dos micronutrientes em clientes depressivos porque acredita-se que esses micronutrientes em quantidades menores, como, selênio, zinco, ferro, magnésio, vitamina B12 e ácido fólico estão vinculados à fisiopatologia da depressão, visto que são essenciais para o funcionamento adequado do sistema nervoso e demais sistemas [24].

A deterioração e o surgimento dos sintomas depressivos em idosos estão associados a diminuição da ingestão alimentar no decorrer do envelhecimento. Podese ter distúrbios na absorção, transporte e até mesmo do metabolismo de nutrientes, caracterizando fatores que podem levar a déficit nutricional, em especial o folato e vitamina B12. Dessa forma, os sintomas depressivos estão associados aos níveis baixos de folato e vitamina B12 [25].

Diferente do autor supracitado, pesquisas indicam que a depressão é considerada um fator que colabora com a perda de peso em idosos. Porém, a relação entre desnutrição e depressão ainda não está clara. No entanto, na terceira idade, a desnutrição, como condição física, pode levar a depressão baseada na sua fragilidade psicológica. E da mesma forma, a depressão pode ocasionar a desnutrição, que pode levar a várias doenças físicas. Um exemplo das causas de depressão que induz a desnutrição e problemas psicológicos em idosos, está baseada na quebra de ligações sociais e experiência de perda [26].

A depressão é uma patologia que influencia de forma direta o apetite e leva a redução do peso e de acordo com outras pesquisas citadas descreve que essa diminuição do peso corporal é um preditor de elevação dos sintomas da depressão na terceira idade [22].

A desnutrição pode estar relacionada com a redução do apetite, diminuição da habilidade de reconhecer os alimentos e manipulação, dificuldades de mastigação e deglutição, considerando o comprometimento cognitivo como um fator de risco para a desnutrição. A redução da mobilidade e abandono social são fatores que podem ocasionar a desnutrição também, alterando seu prognóstico e elevando a mortalidade $[21,22]$. 
A relação entre a desnutrição e depressão é biologicamente possível, porque diversas vias como inflamação, estresse oxidativo e níveis reduzidos de antioxidantes, mantém o papel subjacente de vários nutrientes na explicação do mecanismo de depressão. Ainda não se sabe se realmente a depressão pode levar à redução do apetite e consequentemente à desnutrição. Em contrapartida, a má nutrição pode piorar a condição da depressão [18,27].

\section{Conclusão}

No presente artigo é possível observar que a longevidade está cada vez maior e como vem sendo acompanhada por doenças incapacitantes que interferem diretamente na qualidade de vida da população idosa. É o caso da depressão, que ao longo dos anos é vista como uma patologia que é esperada nos idosos, sendo na verdade uma enfermidade que precisa ser avaliada, diagnosticada e tratada. Um aspecto importante observado é que as limitações cognitivas e funcionais levam à perda da autonomia e independência dos idosos e dessa forma muitas habilidades aprendidas ao longo da vida são interferidas.

Através da realização da presente pesquisa, o objetivo proposto foi alcançado ao evidenciar que existe relação entre a desnutrição e depressão, já que diversas patologias possuem a etiologia decorrente do estresse oxidativo por níveis reduzidos de micronutrientes, explicando o papel de vários nutrientes na manutenção do mecanismo de depressão.

Em vista dos argumentos apresentados, é necessário investigar se existe ou não a depressão nos idosos, a fim de avaliar o estado nutricional. Além disso, a desnutrição foi apontada como um dos fatores que acarretam a depressão, por deficiência de nutrientes e vitaminas.

Outro achado importante evidenciado na literatura foi a prevalência de mulheres com depressão e desnutrição em relação aos homens, podendo ser explicada por fatores fisiológicos, hormonais e aspectos socioculturais.

Vale salientar que, apesar da importância do tema, há escassez de artigos que abordem sobre a relação entre a depressão e a desnutrição, sendo poucas as explicações entre os dois aspectos. Dessa maneira, é importante avaliarmos a significância desses dois assuntos correlacionados e como podem interferir na vida da população da terceira idade. Dessa forma, faz-se necessário realizar mais pesquisas e aprimorar o conhecimento sobre o tema, a fim de amenizar os fatores desencadeantes e proporcionar uma longevidade com qualidade. 
1. Ministério da Saúde (BR). Secretaria de Atenção à Saúde. Departamento de Atenção Básica. Envelhecimento e saúde da pessoa idosa. Cadernos de Atenção Básica [Internet]. 2006 [cited 2021 Aug 25];19(1). Available from: https://bvsms.saude.gov.br/bvs/publicacoes/evelhecimento_saude_pessoa_idosa.pdf

2. Miranda GMD, Mendes ACG, Silva AL. Population aging in Brazil: current and future social challenges and consequences. Rev Bras Geriatr Gerontol 2016;19(3):507-19. doi: 10.1590/1809-98232016019.150140

3. Nóbrega IRAP, Leal MCC, Marques APO, Vieira JCM. Fatores associados à depressão em idosos institucionalizados: revisão integrativa. Saúde Debate 2015;39(105):536-50. doi: 10.1590/0103-110420151050002020

4. Marques JFS, Sá SC, Filho WF, Santo LRE, Prince KA, Oliveira MVM. Transtorno depressivo maior em idosos não institucionalizados atendidos em um centro de referência. Arq Ciênc Saúde 2017;24(4):20-4. doi: 10.17696/2318-3691.24.4.2017.804

5. Ramos FP, Silva SC, Freitas DF, Gangussu LMB, Bicalho AH, Sousa BVO, et al. Fatores associados à depressão em idosos. Revista Eletrônica Acervo Saúde 2019;(19). doi: 10.25248/reas.e239.2019

6. Frade J, Barbosa P, Cardoso S, Nunes C. Depressão nos idosos: sintomas em indivíduos institucionalizados e não-institucionalizados. Rev Enf Ref 2015;4(4):41-9. doi: 10.12707/RIV14030

7. Apóstolo JLA, Campos EMB, Reis IAC, Henriques SJ, Correia CAV. Capacidade de rastreio da escala de depressão geriátrica com 10 e 5 itens. Rev Enf Ref 2018;4(16):29-40. doi: 10.12707/RIV17062

8. Hellwing N, Munhoz TN, Tomasi E. Sintomas depressivos em idosos: estudo transversal de base populacional. Ciênc Saúde Coletiva 2016;21(11):3575-84. doi: 10.1590/1413-812320152111.19552015

9. Scattolin MAA, Avelar NG, Toledo JCF, Yamamoto FW, Alves ER, Neto VSD. Avaliação Nutricional de Idosos Internados no CHS: perfíl nutricional à internação e correlação com escala de depressão e mini-mental. Rev Fac Ciênc Méd Sorocaba [Internet]. 2005 [cited 2021 Aug 25];7(1):15-20. Available from: https://revistas.pucsp.br/RFCMS/article/view/321/pdf

10. Santos $C A$, Ribeiro $A Q$, Rosa COB, Ribeiro RCL. Depressão, déficit cognitivo e fatores associados à desnutrição em idosos com câncer. Ciênc Saúde Coletiva 2015;20(3):751-60. doi: 10.1590/1413-81232015203.06252014

11. Fidelix MSP, Santana AFF, Gomes JR. Prevalência de desnutrição hospitalar em idosos. Revista da Associação Brasileira de Nutrição [Internet]. 2013 [cited 2021 Aug 25];5(1):60-68. Disponível em: https://www.rasbran.com.br/rasbran/article/view/8/10

12. Valença JM, Andrade KL. Desnutrição associada à depressão em idosos hospitalizados. Geriatria \& Gerontologia [Internet]. 2011 [cited 2021 Aug 25];5(1):14-18. Disponível em: https://cdn.publisher.gn1.link/ggaging.com/pdf/v5n1a04.pdf 
13. Barbosa RL, Silva TDCS, Santos MF, Carvalho FR, Marques RVDA, Junior EMM. Perfil sociodemográfico e clínico dos idosos de um centro de convivência. Rev Kairós 2018;21(2):357-73. doi: 10.23925/2176-901X.2018v21i2p357-373

14. Azevedo LC, Fenilli M, Neves L, Almeida CB, Maura BF, Farias MB, et al. Principais fatores da mini-avaliação nutricional associada a alterações nutricionais de idosos hospitalizados. Arq Catarin Med [Internet]. 2007 [cited 2021 Aug 25];36(3):7-14. Available from: https://www.rasbran.com.br/rasbran/article/view/337

15. Souza MT, Silva MD, Carvalho R. Revisão integrativa: o que é e como fazer. Einstein 2010;8(1):102-106. doi: 10.1590/s1679-45082010rw1134

16. Galvão CM. Níveis de evidência. Acta Paul Enferm [Internet]. 2006;19(2). doi: 10.1590/S0103-21002006000200001

17. Galvão TF, Pansani TSA, David H. Principais itens para relatar revisões sistemáticas e meta-análises: A recomendação PRISMA. Epidemiol Serv Saúde 2015;24(2). doi: 10.5123/S1679-49742015000200017

18. Ahmadi SM, Mohammadi MR, Mostafavi SA, Keshavarzi S, Kooshesh SMA, Joulaei H, et al. Dependence of the geriatric depression on nutritional status and anthropometric indices in elderly population. Iran J Psychiatry [Internet]. 2013 [cited 2021 Aug 25];8(2):92-6. Available from: https://www.ncbi.nlm.nih.gov/pmc/articles/PMC3796300/

19. Masón DFC, Suasnavas GFC, Suasnavas VAC. Relación entre desnutrición y depresión en mujeres adultas mayores en la consulta de un hospital de Quito, 2017. Cambios Rev Méd [Internet]. 2018[cited 2021 Aug 25];17(2):5-11. Available from: https://pesquisa.bvsalud.org/portal/resource/pt/biblio-1005224

20. Damo CC, Doring M, Alves ALS, Portella MR. Risk of malnutrition and associated factors in institutionalized elderly persons. Rev Bras Geriatr Gerontol 2018;21(6):711-7. doi: 10.1590/1981-22562018021.180152

21. Santos CA, Ribeiro AQ, Rosa COB, Ribeiro RCL. Depressão, déficit cognitivo e fatores associados à desnutrição em idosos com câncer. Ciênc Saúde Coletiva 2015;20(3):751-60. doi: 10.1590/1413-81232015203.06252014

22. Cruz EP, Sanchéz DCL, Esteves MRM. Asociación entre desnutrición y depresión en el adulto mayor. Nutr Hosp 2014;29(4):901-6. doi: 10.3305/nh.2014.29.4.7228

23. Silva JL, Marques APO, Leal MCC, Alencar DL, Melo EMA. Fatores associados à desnutrição em idosos institucionalizados. Rev Bras Geriatr Gerontol 2015;18(2):44351. doi: $10.1590 / 1809-9823.2015 .14026$

24. Ibarra O, Gili M, Roca M, Vives M, Serrano MJ, Pareja A, et al. The Mediterranean diet and micronutrient levels in depressive patients. Nutr Hosp 2015;31(3):1171-5. doi: 10.3305/nh.2015.31.3.8124

25. Noronha MB, Cunha NA, Araújo DA, Abrunhosa SF, Rocha AD, Amaral TF. Undernutrition, serum vitamin B12, folic acid and depressive symptoms in older adults. Nutr Hosp 2015;31(1):354-61. doi: 10.3305/nh.2015.32.1.8880 
26. Keshavarzi S, Ahmadi SM, Lankarani KB. The impact of depression and malnutrition on health-related quality of life among the elderly Iranians. Glob J Health Sci 2015;7(3):161-70. doi: 10.5539/gjhs.v7n3p161

27. Ghimire S, Baral BK, Pokhrel BR, Pokhrel A, Acharya A, Amatya D, et al. Depression, malnutrition, and health-related quality of life among Nepali older patients. BMC Geriatrics 2018;18(191):1-15. doi: 10.1186/s12877-018-0881-5 